\title{
Oligodendrocyte precursors induce early blood-brain barrier opening after white matter injury
}

\author{
Ji Hae Seo, ${ }^{1,2}$ Nobukazu Miyamoto, ${ }^{1}$ Kazuhide Hayakawa, ${ }^{1}$ Loc-Duyen D. Pham, ${ }^{1}$ \\ Takakuni Maki, ${ }^{1}$ Cenk Ayata, ${ }^{3}$ Kyu-Won Kim, ${ }^{2,4,5}$ Eng H. Lo, ${ }^{1}$ and Ken Arai ${ }^{1}$
}

\begin{abstract}
${ }^{1}$ Neuroprotection Research Laboratory, Departments of Radiology and Neurology, Massachusetts General Hospital and Harvard Medical School, Boston, Massachusetts, USA. ${ }^{2}$ NeuroVascular Coordination Research Center, College of Pharmacy, Seoul National University, Seoul, Republic of Korea. ${ }^{3}$ Neurovascular Research Laboratory, Department of Radiology, Massachusetts General Hospital and Harvard Medical School, Boston, Massachusetts, USA. ${ }^{4}$ Department of Molecular Medicine and Biopharmaceutical Sciences, Graduate School of Convergence Science and Technology, and ${ }^{5}$ Department of Internal Medicine, and Innovative Research Institute for Cell Therapy, Seoul National University, Seoul, Republic of Korea.
\end{abstract}

\begin{abstract}
Oligodendrocyte precursor cells (OPCs) are thought to maintain homeostasis and contribute to long-term repair in adult white matter; however, their roles in the acute phase after brain injury remain unclear. Mice that were subjected to prolonged cerebral hypoperfusion stress developed white matter demyelination over time. Prior to demyelination, we detected increased MMP9 expression, blood-brain barrier (BBB) leakage, and neutrophil infiltration in damaged white matter. Notably, at this early stage, OPCs made up the majority of MMP9-expressing cells. The standard MMP inhibitor GM6001 reduced the early BBB leakage and neutrophil infiltration, indicating that OPC-derived MMP9 induced early BBB disruption after white matter injury. Cellculture experiments confirmed that OPCs secreted MMP9 under pathological conditions, and conditioned medium prepared from the stressed OPCs weakened endothelial barrier tightness in vitro. Our study reveals that OPCs can rapidly respond to white matter injury and produce MMP9 that disrupts the BBB, indicating that OPCs may mediate injury in white matter under disease conditions.
\end{abstract}

\section{Introduction}

In adult brains, oligodendrocyte precursor cells (OPCs) are thought to maintain homeostasis and mediate long-term repair in white matter after injury and disease (1). In response to demyelination signals, OPCs proliferate, migrate, and rapidly fill in the damaged area, followed by differentiating into mature oligodendrocytes to form and restore myelin sheaths $(2,3)$. The increase in OPC numbers is observed at the margins of infarcts after focal ischemia (4). Moreover, the OPC proliferation rate is also increased in ischemic white matter lesions after prolonged cerebral hypoperfusion (5). Hence, the activation of OPCs is supposed to represent a protective response in damaged or diseased brain.

Recently, however, reactive glia are now recognized to mediate complex mechanisms, including both beneficial and deleterious effects after brain injury and neurodegeneration $(6,7)$. For example, depending on context, astrocytes can either promote neuroplasticity (8) or secrete inhibitory matrix molecules that inhibit axons (9). Similarly, microglia are now known to release both prorecovery and neurotoxic factors (10-12). Is it possible that OPCs may also share these biphasic properties? Beyond the standard role of contributing to oligodendrogenesis and remyelination, OPCs can also release multiple factors to modulate neighboring cells and the microenvironment $(1,13)$. These intercellular signals may be especially important, since perturbations in the blood-brain barrier (BBB) are known to be a critical part of white matter pathology in a wide range of CNS disorders $(14,15)$. In this study, therefore, we asked whether OPCs can somehow influence BBB function after white matter injury.

Conflict of interest: The authors have declared that no conflict of interest exists. Citation for this article: J Clin Invest. 2013;123(2):782-786. doi:10.1172/JCI65863.

\section{Results and Discussion}

Mice were subjected to prolonged cerebral hypoperfusion (16). As expected, after 7 days, they developed white matter injury and demyelination (Figure 1A and Supplemental Figures 1 and 2; supplemental material available online with this article; doi:10.1172/JCI65863DS1). However, even before demyelination occurred, BBB leakage (Supplemental Figure 3) and neutrophil infiltration (Supplemental Figure 4) were observed. Because the gelatinases, MMP2 and -9 , are known to mediate BBB injury $(17,18)$, we examined MMP2/9 responses in our model. During the early phase of BBB leakage at 3 days, white matter MMP9 expression, but not MMP2 expression, was increased (Figure 1B and Supplemental Figure 5), mostly in OPCs (Figure 1, C and E). During later time points, at 7 or 14 days, expression patterns shifted and a secondary expression of MMP9 occurred in cerebral endothelial cells (Figure 1, D and E). No other glial cell types (mature oligodendrocytes, astrocytes, microglia) appeared to produce MMP9 in this model of cerebral hypoperfusion and chronic hypoxic stress (Supplemental Figure 6). Moreover, no significant MMP9 increases were observed in blood neutrophils nor plasma at day 3 in our model (Supplemental Figure 7). Importantly, MMP9-expressing OPCs were located close to cerebral endothelial cells (Supplemental Figure 8), and indeed, OPCs existed near the BBB leakage areas at the acute phase of white matter injury (Figure $1 \mathrm{~F}$ ).

To confirm that OPCs have a capacity of releasing MMP9 under pathologic conditions, we prepared primary OPC cultures from neonatal rat brains (Supplemental Figure 9). Gelatin zymography showed that OPCs did not produce MMP9 under normal conditions, but after treatment with nonlethal levels of the inflammatory cytokine IL-1 $\beta$, MMP9 secretion was markedly increased 
A

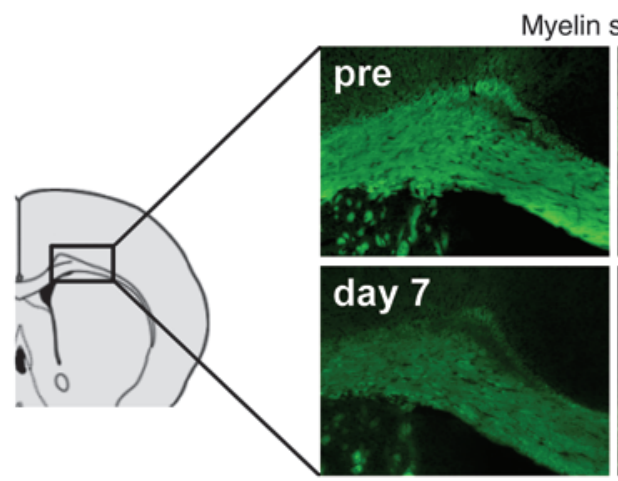

C
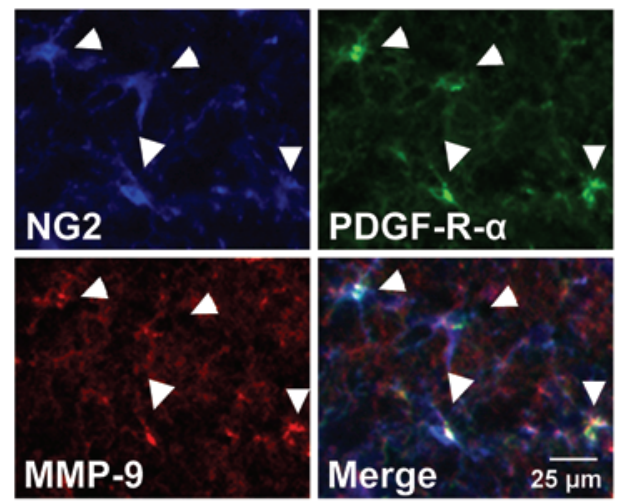

$\mathbf{F}$

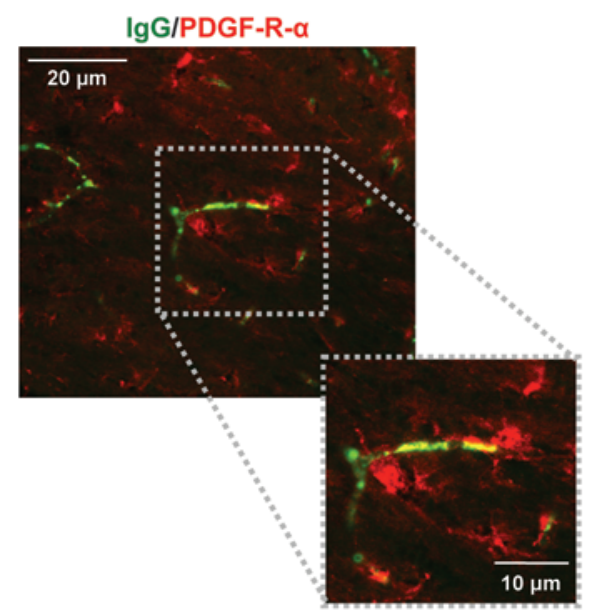

B

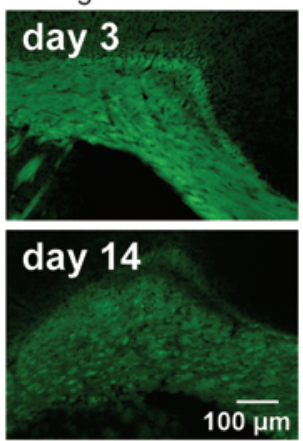

D
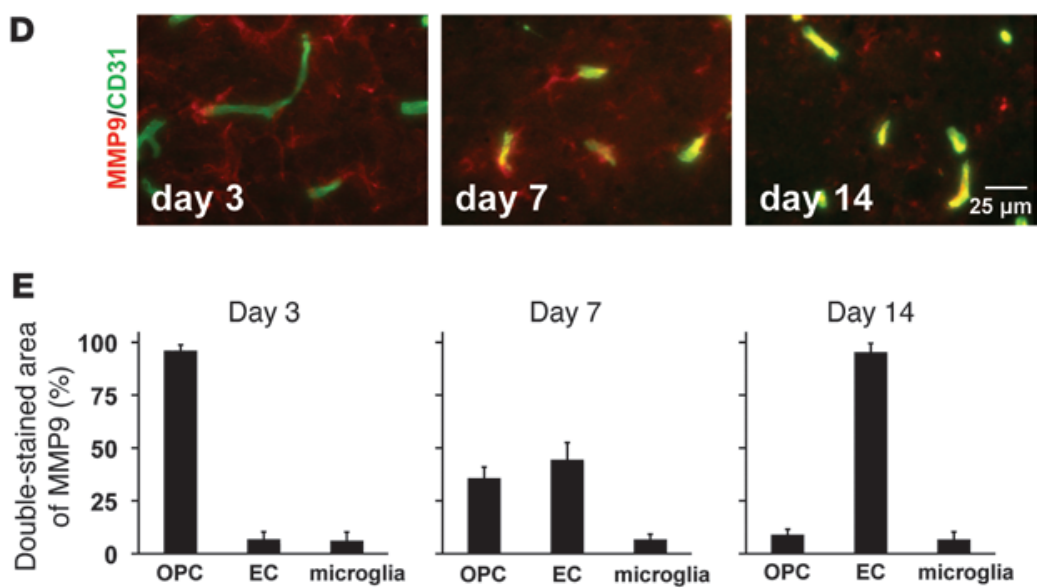

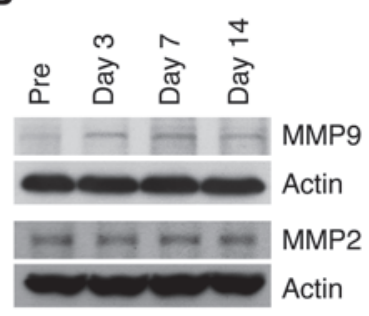

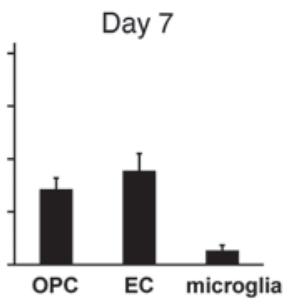

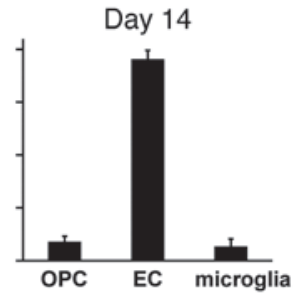

Figure 1

OPCs and MMP9 under white matter pathology. (A) Cerebral prolonged hypoperfusion stress-induced demyelination in the mouse corpus callosum. $n=5$. Quantitative data are shown in Supplemental Figure 1. (B) In our white matter injury model, MMP9 but not MMP2 was increased in the white matter. (C-E) At day 3, most MMP9 signals were observed in NG2/PDGF-R- $\alpha$-positive OPCs. But at later time points, at days 7 and 14, CD31-positive cerebral endothelial cells (EC) were colocalized with MMP9 signals. $n=5$. (F) Notably, OPCs (PDGF-R- $\alpha$ ) existed around BBB leakage areas (lgG) at day 3.

(Figure 2A and Supplemental Figure 10). Next, we performed medium transfer experiments (Figure 2B) to ask whether MMP9 from stimulated OPCs could perturb endothelial BBB function. Conditioned medium from normal OPCs did not alter endothelial function, but conditioned medium from IL-1 $\beta$-treated OPCs degraded the tight-junction protein $\mathrm{ZO}-1$ in cerebral endothelial cells without affecting cell survival (Figure 2, C-E, and Supplemental Figure 11). Consistent with this effect on tight junctions, conditioned medium from IL- $1 \beta$-stimulated OPCs significantly increased endothelial permeability and neutrophil transmigration (Figure 2, F and G, and Supplemental Figure 12). This effect was dependent on MMPs; the broad-spectrum MMP inhibitor GM6001 ameliorated these perturbations in endothelial permeability and neutrophil transmigration (Figure 2, F and G).
Thus far, our in vivo and in vitro findings suggest that stimulated OPCs release MMP9, which degrades the BBB in white matter. If so, does this potentially represent a therapeutic target that interrupts white matter disease at an early stage, thus preventing further damage and downstream inflammation and demyelination? To test this hypothesis, mice were subjected to cerebral hypoperfusion and treated with either vehicle or GM6001 immediately after surgery and on day 2 after hypoperfusion onset (Figure 3A). GM6001 has a short half-life, so this treatment protocol limits MMP inhibition to only the early phase, when MMP9 is expressed in OPCs. As expected, BBB leakage and neutrophil infiltration on day 3 were reduced by the GM6001 treatments (Figure 3, B-D, and Supplemental Figure 13). Importantly, inhibition of the early MMP9 phase in OPCs also prevented the secondary expression of 
A

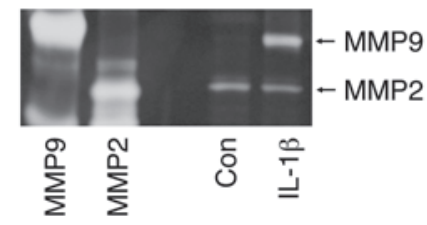

B

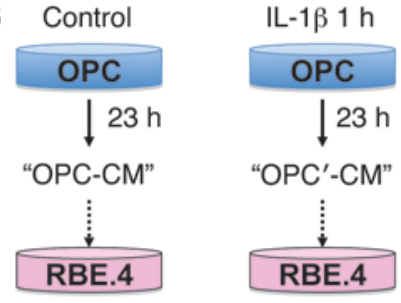

C

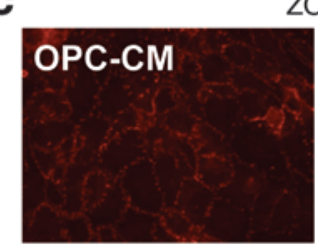

$\mathrm{ZO}-1$

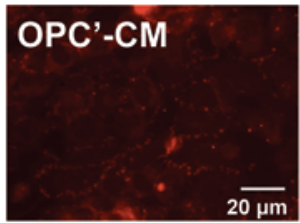

D

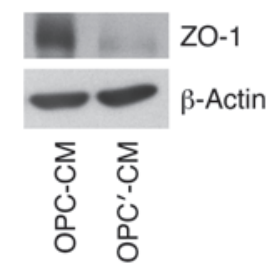

E

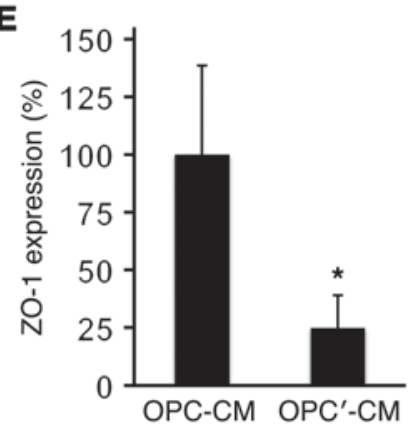

F

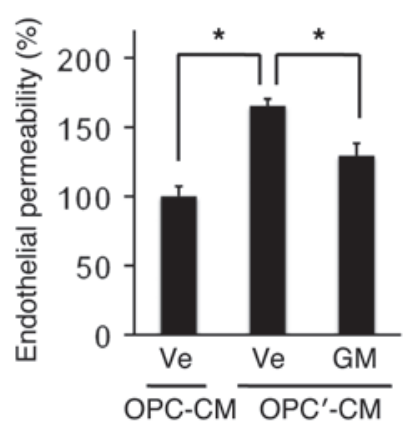

G

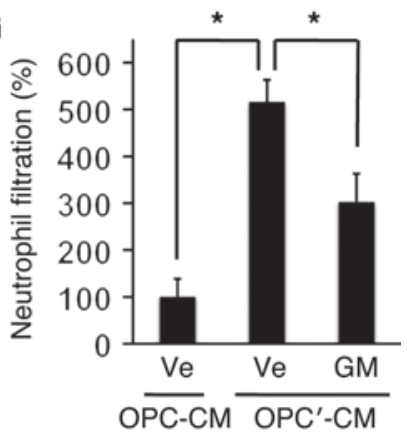

Figure 2

OPC cultures and in vitro BBB model. (A) Gelatin zymography showed that MMP9 but not MMP2 was secreted from cultured rat OPCs by treatment with IL-1 $\beta$ (1-hour treatment at $100 \mathrm{ng} / \mathrm{ml}$ followed by 23-hour incubation under normal culture conditions). (B) We prepared conditioned medium from normal OPCs (OPC-CM) or IL-1 $\beta$-treated OPCs (OPC'-CM), and then added them to cerebral endothelial RBE.4 cells. (C-E) Twenty-four hours after treatment with OPC'-CM degraded the tight-junction protein ZO-1 in RBE.4 cells. $n=3$. ${ }^{*} P<0.05$. (F and G) Compared with OPC-CM, OPC'-CM increased both endothelial permeability and neutrophil filtration. Importantly, BBB breakdown was reduced by cotreatment with MMP inhibitor GM6001 (10 $\mu \mathrm{M})$. Ve, vehicle; GM, GM6001. $n=3$. * $P<0.05$.

MMP9 in cerebral endothelium on day 7 (Figure 3E and Supplemental Figure 14) and subsequently the development of white matter injury and demyelination (Figure 3F and Supplemental Figure 15) as well as cognitive deficits (Supplemental Figure 16) in this model of cerebral hypoperfusion. Those data indicate that OPC-derived MMP9 in the acute phase may trigger further secondary cascades of cerebrovascular damage in white matter.

After brain injury and disease, progenitor/precursor cells in the adult brain are thought to be recruited as part of an endogenous response that helps compensate for lost brain function $(19,20)$. For example, OPCs can be triggered to become mature oligodendrocytes (or cortical projection neurons) after white matter injury $(1,21)$. But beyond this expected beneficial role, our current study suggests that under some conditions, OPCs can rapidly respond to white matter injury and produce MMP9 that appears to open the $\mathrm{BBB}$ and trigger secondary cascades of cerebrovascular injury and demyelination (Figure 3G).

Taken together, our cell and in vivo data suggest that OPCs in adult brain are not merely precursor cells for white matter remodeling, but may also surprisingly contribute to injury and demyelination under some injury or disease conditions. Nevertheless, there are a few issues that warrant further studies. First, we only focus on OPCs, but other precursor/progenitor cells might also be important. Roles of neuronal or endothelial precursor cells and pericytes on $\mathrm{BBB}$ dysregulation should be examined in future studies. Second, although OPCs contribute to the early BBB opening after injury in white matter, what cell types contribute in the gray matter? Comparing mechanisms between gray and white matter will be very important. Third, we only focus on the roles of gelatinases (MMP2 and -9). They are well-known proteinases that break down BBB after brain injury, but our current pharmacological approach is somewhat limited to the broad-spectrum MMP inhibitor GM6001, which can inhibit MMP1 and -8 in addition to MMP2 and -9. Future studies should carefully examine roles of the larger MMP network in the white matter pathophysiology. Finally, we need to assess the deleterious function of OPCs in other diseased models. Previous study has demonstrated that MMP9 is expressed in endothelial cells at early time points after stroke (22). Hence, in other brain diseases, OPCs may cooperate with other cell types to induce vascular dysfunction at an early injury phase.

Dynamic interactions among neuronal, glial, and vascular cells contribute to brain homeostasis, while abnormal cell-cell signaling leads to BBB dysfunction in various brain diseases (23). Notably, white matter pathophysiology is an important aspect in those diseases (24). Because early GM6001 treatment prevented demyelination and cognitive deficits in our white matter injury model, future studies are warranted to investigate drugs that target OPCs for white matter diseases such as stroke or vascular dementia. In conclusion, our study suggests that OPCs can play a surprisingly deleterious role in cerebrovascular injury and demyelination in white matter.

\section{Methods}

Prolonged cerebral hypoperfusion stress by bilateral common carotid artery stenosis. Male C57BL/ 6 mice (10 to 12 weeks old, 24 to $29 \mathrm{~g}$ ) were anesthetized with $4.0 \%$ isoflurane and maintained on $1.5 \%$ isoflurane in $70 \% \mathrm{~N}_{2} \mathrm{O}$ and $30 \%$ $\mathrm{O}_{2}$ using a small-animal anesthesia system. Through a midline cervical incision, both common carotid arteries were exposed. A micro-coil with a diameter of $0.18 \mathrm{~mm}$ (Sawane Spring Co.) was applied to the bilateral common carotid artery. 
A

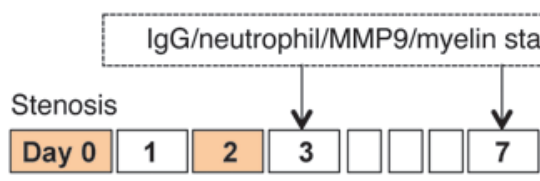

Vehicle or $10 \mathrm{mg} / \mathrm{kg} \mathrm{GM6001} \mathrm{ip}$
C

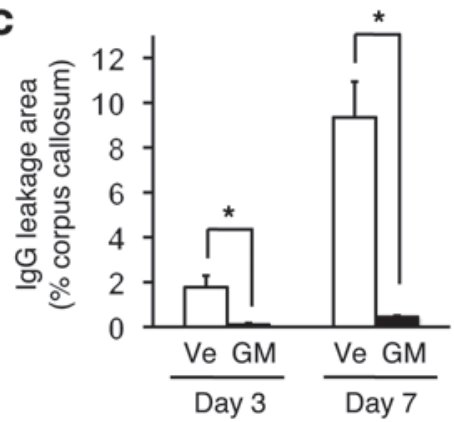

D

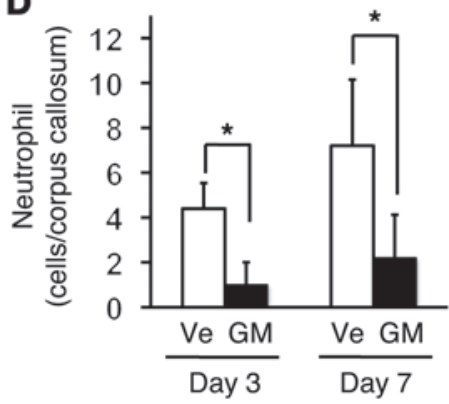

B
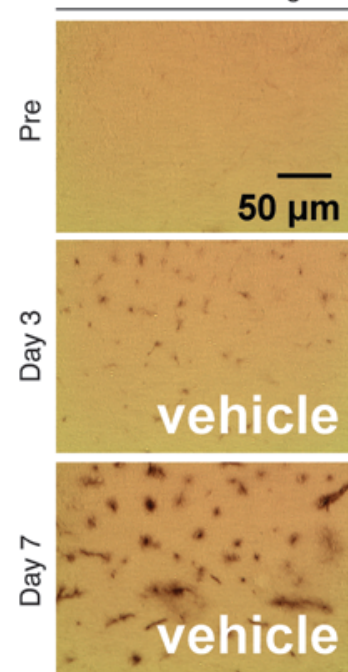

IgG staining

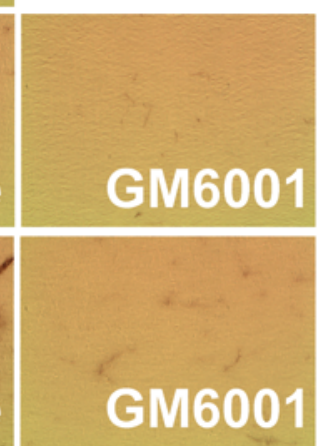

E

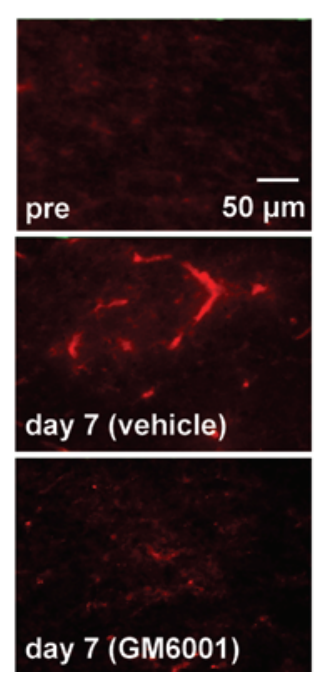

F
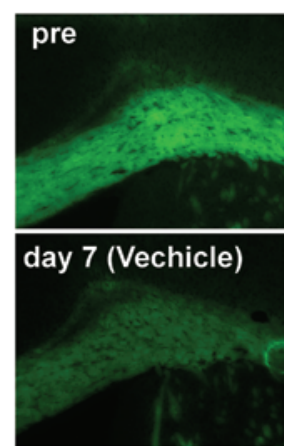

day 7 (GM6001)

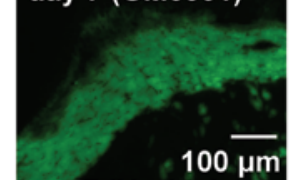

G

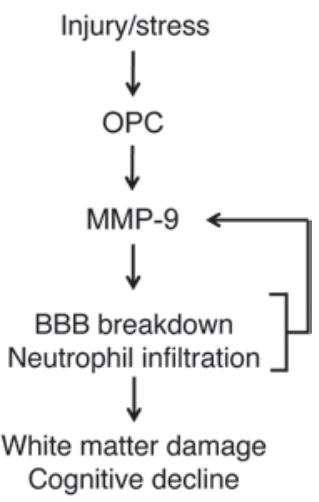

Figure 3

OPCs and BBB function under white matter pathology. (A) Schematics for in vivo experiments using mouse white matter injury model. (B-D) Treatments with GM6001 blocked both BBB leakage and neutrophil infiltration at days 3 and 7 after white matter injury by prolonged cerebral hypoperfusion. Representative images for neutrophil staining are shown in Supplemental Figure 13. $n=5$. ${ }^{*} P<0.05$. (E and F) GM6001 also inhibited MMP9 production in endothelium as well as the development of white matter injury at day $7 . n=5$. Quantitative data for MMP9 production and myelin density are shown in Supplemental Figures 14 and 15, respectively. (G) Schematics for the role of OPCs under acute phase after white matter injury; OPC secretes MMP9 to trigger BBB disruption leading to BBB breakdown and neutrophil infiltration.

Myelin staining. Mouse coronal sections of $12-\mu \mathrm{m}$ thicknesses were prepared on glass slides using cryostat. Myelins were visualized using FluoroMyelin green fluorescent myelin stain kit (Molecular probes).

IgG staining. After PFA fixation, $12-\mu \mathrm{m}$-thick brain sections were incubated in $3 \% \mathrm{H}_{2} \mathrm{O}_{2}$, followed by blocking with $10 \%$ bovine serum albumin (Sigma-Aldrich) in PBS. Then the sections were incubated overnight at $4^{\circ} \mathrm{C}$ with antibody against donkey anti-mouse IgG (1:200; Jackson Immunoresearch Laboratories). Immunoreactivity was visualized using the avidin-biotin complex method (ABC Staining Kits; Pierce Biotechnology) or fluorescence-conjugated streptavidin.

Immunohistochemistry. Mouse brains were taken out after perfusion with PBS ( $\mathrm{pH}$ 7.4) and then quickly frozen using liquid nitrogen. Coronal sections of $16-\mu \mathrm{m}$ thicknesses were cut on cryostat at $-20^{\circ} \mathrm{C}$ and collected on glass slides. Sections were fixed by $4 \% \mathrm{PFA}$ and rinsed 3 times in PBS ( $\mathrm{pH}$ 7.4). After blocking with $3 \% \mathrm{BSA}$, sections were incubated at $4^{\circ} \mathrm{C}$ overnight in PBS $/ 0.1 \%$ Tween $/ 0.3 \%$ BSA solution containing primary antibodies. Then sections were washed and incubated with secondary antibodies with fluorescence conjugations at room temperature for 1 hour.

Western blotting. Lysates from the corpus callosum region were prepared in Pro-PREPTM Protein Extraction Solution (Boca Scientific). Samples were heated with equal volumes of SDS sample buffer (Novex) and $10 \mathrm{mM}$ DTT at $95^{\circ} \mathrm{C}$ for 5 minutes; then each sample ( $20 \mu \mathrm{g}$ per lane) was loaded onto $4 \%-20 \%$ Tris-glycine gels. After electrophoresis and transferring to polyvinylidene difluoride membranes (Novex), the membranes were blocked in Brockace (AbD Serotec) for 60 minutes at room temperature. Membranes were then incubated overnight at $4^{\circ} \mathrm{C}$ in Brockace solution containing primary antibodies, followed by incubation with peroxidase-conjugated secondary antibodies.

Cell culture. OPCs were prepared from cerebral cortices of 1- to 2-day-old Sprague-Dawley rats according to our previous study (25). OPCs were cultured in Neurobasal Media (Invitrogen) containing glutamine, 1\% penicillin/streptomycin, $10 \mathrm{ng} / \mathrm{ml}$ PDGF, $10 \mathrm{ng} / \mathrm{ml} \mathrm{FGF}$, and 2\% B27 supplement onto poly-dl-ornithine-coated plates. Rat brain microendothelial cell lines (RBE.4) were cultured in EGM-2MV containing a EGM-2MV Single Quots kit onto collagen-coated flasks. Leukocytes were prepared from mouse peritoneal cavity. Twenty-four hours later, nonadherent cells were collected as a leukocyte population, then cultured in RPMI medium containing $10 \%$ fetal bovine serum and $1 \%$ penicillin/streptomycin.

Preparation of OPC-conditioned medium. One hour after treatment with IL-1 $\beta$, OPC cultures were washed with PBS to remove IL-1 $\beta$ and maintained in Neurobasal medium for 23 hours. Culture medium was then 
collected and centrifuged at $10,000 \mathrm{~g}$ for 5 minutes at $4^{\circ} \mathrm{C}$ to remove cells and debris. The $\mathrm{OPC}$-conditioned medium was stored at $-80^{\circ} \mathrm{C}$ until use.

Gelatin zymography. The collected conditioned medium was concentrated using Microcon (Millipore) with a $10-\mathrm{kDa}$ pore diameter cutoff, then each sample was mixed with equal amounts of SDS sample buffer (Novex) and electrophoresed on 10\% SDS-polyacrylamide gels (Novex) containing $1 \mathrm{mg} / \mathrm{ml}$ gelatin as the protease substrate. Following electrophoresis, gels were placed in $2.7 \%$ Triton X-100 for 1 hour to remove SDS and then incubated for 16 hours at $37^{\circ} \mathrm{C}$ in Novex's developing buffer on a rotary shaker. After incubation, gels were stained in 30\% methanol, $10 \%$ acetic acid, and $0.5 \% \mathrm{w} / \mathrm{v}$ coomassie brilliant blue for 1 hour followed by destaining. Mixed human MMP2 and MMP9 standards (Chemicon) were used as positive controls.

Immunocytochemistry. After cells were confluent, they were washed with ice-cold PBS ( $\mathrm{pH} 7.4$ ), followed by 4\% PFA for 15 minutes. After being further washed 3 times in PBS, they were incubated with 3\% BSA in PBS for 1 hour. Then cells were incubated with primary antibody against ZO-1 (1:200; Invitrogen) at $4{ }^{\circ} \mathrm{C}$ overnight. After washing with PBS, they were incubated with secondary antibodies conjugated with fluorescein isothiocyanate for 1 hour at room temperature. Finally, nuclei were counterstained with DAPI.

In vitro endothelial permeability assay. Permeability across the endothelial cell monolayer was measured by using type I collagen-coated Transwell units ( $6.5 \mathrm{~mm}$ diameter, $3.0 \mu \mathrm{m}$ pore size polycarbonate filter; Corning). After RBE.4 cells became confluent on the Transwell, OPC-conditioned medium was treated for 24 hours. Then, fluorescein isothiocyanate-labeled dextran (molecular weight, 40,000) was added to the upper chamber. After incubation for 10 minutes, $100 \mu \mathrm{l}$ of sample from the lower compartment was measured for fluorescence at $620 \mathrm{~nm}$ when excited at $590 \mathrm{~nm}$ with a spectrophotometer.

In vitro neutrophil filtration assay. Neutrophil transmigration assay was performed using Cytoselect 24-well Migration Assay Kit (Cell Biolabs) according to the manufacturer's instructions. Briefly, cell-culture inserts (3- $\mu \mathrm{m}$ pore size) were used to form dual compartments in a 24-well culture plate. When the RBE.4 cells were confluent in Transwell, freshly isolated mouse neutrophils were loaded on the RBE. 4 monolayer. Neutrophils were allowed to migrate for 24 hours at $37^{\circ} \mathrm{C}$ and $5 \% \mathrm{CO}_{2}$. The neutrophils that had crossed the RBE.4 layer were collected from the lower compartment and stained and quantified.

Please see Supplemental Methods and Supplemental Figures for detailed experimental procedures and additional data.

Statistics. Quantitative data were analyzed by using either 2-tailed $t$-tests or ANOVA, followed by Tukey's honestly significant difference tests. Data are expressed as mean $\pm \mathrm{SD}$. A value of $P<0.05$ was considered significant.

Study approval. All experiments were performed following protocols approved by the Massachusetts General Hospital Institutional Animal Care and Use Committee in accordance with the National Institutes of Health Guide for the Care and Use of Laboratory Animals.

\section{Acknowledgments}

This work was supported in part by the Deane Foundation, the American Heart Association, the NIH, the National Research Foundation of Korea, the World Class University Program, and the Global Research Laboratory Program.

Received for publication October 3, 2012, and accepted in revised form November 1, 2012.

Address correspondence to: Ken Arai or Eng H. Lo, Neuroprotection Research Laboratory, MGH East 149-2401, Charlestown, Massachusetts 02129, USA. Phone: 617.724.9503; Fax: 612.726.7830; E-mail: karai@partners.org (K. Arai). Phone: 617.726.4043; Fax: 612.726.7830; E-mail: lo@helix.mgh.harvard.edu (E.H. Lo).
1. Nishiyama A, Komitova M, Suzuki R, Zhu X. Polydendrocytes (NG2 cells): multifunctional cells with lineage plasticity. Nat Rev Neurosci. 2009;10(1):9-22.

2. Gensert JM, Goldman JE. Endogenous progenitors remyelinate demyelinated axons in the adult CNS Neuron. 1997;19(1):197-203.

3. Redwine JM, Armstrong RC. In vivo proliferation of oligodendrocyte progenitors expressing PDGFalphaR during early remyelination. J Neurobiol. 1998;37(3):413-428

4. Tanaka K, et al. Activation of NG2-positive oligodendrocyte progenitor cells during post-ischemic reperfusion in the rat brain. Neuroreport. 2001;12(10):2169-2174.

5. Miyamoto N, et al. Phosphodiesterase III inhibition promotes differentiation and survival of oligodendrocyte progenitors and enhances regeneration of ischemic white matter lesions in the adult mammalian brain. J Cereb Blood Flow Metab. 2010;30(2):299-310.

6. Fitch MT, Silver J. CNS injury, glial scars, and inflammation: Inhibitory extracellular matrices and regeneration failure. Exp Neurol. 2008;209(2):294-301.

7. Myer DJ, Gurkoff GG, Lee SM, Hovda DA, Sofroniew MV. Essential protective roles of reactive astrocytes in traumatic brain injury. Brain. 2006;129(pt 10):2761-2772.

8 . Xin $\mathrm{H}$, et al. Increasing tPA activity in astrocytes induced by multipotent mesenchymal stromal cells facilitate neurite outgrowth after stroke in the mouse. PLoS One. 2010;5(2):e9027.

9. Silver J, Miller JH. Regeneration beyond the glial scar. Nat Rev Neurosci. 2004;5(2):146-156.

10. Ransohoff RM, Brown MA. Innate immunity in the central nervous system. J Clin Invest. 2012; 122(4):1164-1171.

11. Tremblay ME, Stevens B, Sierra A, Wake H, Bessis A, Nimmerjahn A. The role of microglia in the healthy brain. J Neurosci. 2011;31(45):16064-16069.

12. Yenari MA, Kauppinen TM, Swanson RA. Microglial activation in stroke: therapeutic targets. Neurotherapeutics. 2010;7(4):378-391.

13. Du Y, Dreyfus CF. Oligodendrocytes as providers of growth factors. J Neurosci Res. 2002;68(6):647-654.

14. Yang Y, Rosenberg GA. Blood-brain barrier breakdown in acute and chronic cerebrovascular disease. Stroke. 2011;42(11):3323-3328.

15. Saunders NR, Ek CJ, Habgood MD, Dziegielewska KM. Barriers in the brain: a renaissance? Trends Neurosci. 2008;31(6):279-286.

16. Shibata M, Ohtani R, Ihara M, Tomimoto H. White matter lesions and glial activation in a novel mouse model of chronic cerebral hypoperfusion. Stroke. 2004;35(11):2598-2603.

17. Asahi M, et al. Effects of matrix metalloproteinase- 9 gene knock-out on the proteolysis of blood-brain barrier and white matter components after cerebral ischemia. J Neurosci. 2001;21(19):7724-7732.

18. Rosenberg GA, Estrada EY, Dencoff JE. Matrix metalloproteinases and TIMPs are associated with blood-brain barrier opening after reperfusion in rat brain. Stroke. 1998;29(10):2189-2195.

19. Moskowitz MA, Lo EH, Iadecola C. The science of stroke: mechanisms in search of treatments. Neuron. 2010;67(2):181-198.

20. Hermann DM, Chopp M. Promoting brain remodelling and plasticity for stroke recovery: therapeutic promise and potential pitfalls of clinical translation. Lancet Neurol. 2012;11(4):369-380.

21. Rivers LE, et al. PDGFRA/NG2 glia generate myelinating oligodendrocytes and piriform projection neurons in adult mice. Nat Neurosci. 2008;11(12):1392-1401.

22. Romanic AM, White RF, Arleth AJ, Ohlstein EH, Barone FC. Matrix metalloproteinase expression increases after cerebral focal ischemia in rats: inhibition of matrix metalloproteinase- 9 reduces infarct size. Stroke. 1998;29(5):1020-1030.

23. Zlokovic BV. Neurodegeneration and the neurovascular unit. Nat Med. 2010;16(12):1370-1371.

24. Selnes OA, Vinters HV. Vascular cognitive impairment. Nat Clin Pract Neurol. 2006;2(10):538-547.

25. Arai K, Lo E. An oligovascular niche: cerebral endothelial cells promote the survival and proliferation of oligodendrocyte precursor cells. J Neurosci. 2009;29(14):4351-4356 